\title{
ESCOLITINOS Y PLATIPODINOS (COLEOPTERA: CURCULIONIDAE) ATRAÍDOS A TRAMPAS TIPO NTP-80 EN ZAPOTITLÁN DE LAS SALINAS, PUEBLA, MÉXICO
}

\author{
RobERTo QUEZADA-GARCÍA, ${ }^{1 *}$ EsTEBAN JIMÉNEZ-SÁNCHEZ, ${ }^{2}$ ARMANDo \\ EQUIHUA-MARTÍNEZ ${ }^{3}$ y JORGE PADILLA-RAMÍREZ ${ }^{2}$ \\ ${ }^{1}$ Centre d’Étude de la Forêt (CEF) y Département des Sciences du Bois et de la Forêt, Faculté \\ de Foresterie, Géographie et Géomatique, Université Laval, Québec, Québec, Canada G1V 0A6. \\ *<roberto.quezada-garcia.1@ulaval.ca> \\ ${ }^{2}$ Laboratorio de Zoología FES-Iztacala, Universidad Nacional Autónoma de México. Av. de los \\ barrios \# 1 Tlalnepantla, Estado de México, México C.P. 54090. \\ ${ }^{3}$ Instituto de Fitosanidad, Colegio de Postgraduados Km. 36.5 Carretera México-Texcoco. Estado de \\ México, México. C.P. 56230.
}

Quezada-García, R., Jiménez-Sánchez, E., Equihua-Martínez, A. y Padilla-Ramírez, J. 2014. Escolitinos y platipodinos (Coleoptera: Curculionidae) atraídos a trampas tipo NTP-80 en Zapotitlán de las Salinas, Puebla, México. Acta Zoológica Mexicana (n.s.), 30(3): 625-636.

RESUMEN. Se realizó el estudio de fauna de la escolitinos y platipodinos en una zona semiárida de Zapotitlán de las Salinas, Puebla, México. Se realizaron muestreos mensuales durante un año, de abril de 1998 a marzo de 1999, empleando trampas de tipo NTP-80 cebadas con calamar. El líquido conservador compuesto de ácido acético y alcohol, sirvió como atrayente para estos grupos. Las trampas fueron distribuidas en cinco localidades (cactáceas columnares, mezquital de terrazas, mezquital ribereño, mezquital no fragmentado y una vegetación no alterada). Se capturaron 328 individuos, cinco especies de Scolytinae y una de Platypodinae. Se registraron por primera vez para el estado de Puebla a Premnobius cavipennis Eichhoff, 1878, Xyleborus posticus Eichhoff, 1869, Monarthrum pennatum Wood y Bright, 1992 y Euplatypus parallelus (Fabricius, 1801). La mayor abundancia y riqueza de especies fue observada en el mezquital ribereño y la presencia de estos grupos de Curculionidae de forma abundante al final de la época de lluvias, lo que podría estar asociado con la disponibilidad de recursos en tiempo y espacio. Este estudio contribuye de manera importante al conocimiento de los escolitinos y platipodinos del estado de Puebla, México.

Palabras clave: Premnobius cavipennis, Xyleborus, mezquital, áreas semiáridas, Tehuacán-Cuicatlán.

Quezada-García, R., Jiménez-Sánchez, E., Equihua-Martínez, A. \& Padilla-Ramírez, J. 2014. Scolytinae and Platipodinae (Coleoptera: Curculionidae) attracted to NTP-80 traps in Zapotitlán de las Salinas, Puebla, Mexico. Acta Zoológica Mexicana (n.s.), 30(3): 625-636.

ABSTRACT. A study on Platypodinae and Scolytinae in a semi-arid area from Zapotitlán de las Salinas, Puebla, Mexico was done. Samples were taken monthly from April 1998 to March 1999 using

Recibido: 24/03/2014; aceptado: 24/06/2014. 
NTP-80 carrion traps baited with squid. Conservative liquid composed of acetic acid and alcohol, served as attractant to these groups. The traps were distributed in five locations (columnar cacti, mezquite terraces, riparian mezquite, mezquite unfragmented and undisturbed vegetation). A total of 328 individuals of five species of Scolytinae and one of Platypodinae were collected. Premnobius cavipennis Eichhoff, 1878, Xyleborus posticus Eichhoff, 1869, Monarthrum pennatum Wood \& Bright, 1992 and Euplatypus parallelus (Fabricius, 1801) are recorded for the first time for the state of Puebla. The greatest abundance and species richness of Curculionidae were observed on riparian mezquite, major abundance was observed at the end of the rainy season. Given the environmental degradation in the area, this study makes a contribution to the knowledge of Scolytinae and Platypodinae of Puebla, Mexico.

Key words: Premnobius cavipennis, Xyleborus, mesquite, semiarid areas, Tehuacán-Cuicatlán.

\section{INTRODUCCIÓN}

Las zonas áridas y semiáridas ocupan más del 60\% de la superficie de México; éstas están dividas en cuatro grandes áreas que se extienden desde el norte hasta el centro del país (Rzedowski 1978). Una de estas áreas es el Valle de Tehuacán-Cuicatlán, que ha sido catalogado por algunos autores como una fuente importante de riqueza biológica y social, la cual tiene altos niveles de diversidad y endemismos tanto de animales como de plantas (Dávila et al. 2002, Paredes-Flores et al. 2007). En este valle se encuentra enclavada el área de Zapotitlán de las Salinas, donde los tipos de vegetación representativas son: el cardonal, el mezquital, las cactáceas columnares y el matorral (Oliveros 2000). En esta misma zona Jiménez-Sánchez et al. (2013) encontraron que el tipo de vegetación es un factor importante en la diversidad de coleópteros necrófilos (Silphidae, Scarabaeidae, Trogidae y Staphylinidae), y se observó una asociación importante con el matorral y el mezquital ribereño. Sin embargo, la alarmante degradación de este hábitat natural, resalta la importancia de ampliar el conocimiento de su biodiversidad (Casas et al. 2001).

En particular, la trampa tipo NTP-80 se utiliza para colectar insectos que son comúnmente atraídos a la carroña (Morón \& Terrón 1984), sin embargo, la mezcla de ácido acético y alcohol utilizada como líquido conservador, ha demostrado atraer una diversidad importante de otros grupos que generalmente se asocian a plantas vivas o en descomposición, como ocurre con los escarabajos Nitidulidae y Curculionidae (Morón \& Terrón 1982, 1984, Morón \& López-Méndez 1985, Morón et al. 1986, Deloya et al. 1987, Cejudo-Espinosa \& Deloya 2005, Navarrete-Heredia et al. 2012). Aunque la familia Nitidulidae incluye también especies comunes en las fases tardías de descomposición del cadáver (Gennard 2007). Los representantes de Curculionoidea que se han encontrado en este tipo de trampas son: Brentidae (Apioninae), Dryophthoridae (citado como Rhynchophoridae) y Curculionidae (Entiminae, Molytinae, Cryptorhynchinae) (Muñiz 1998), dentro de esta última también se tienen aquellos de hábitos ambrosiales como las subfamilias Scolytinae y Platypodinae (Equihua 1992, Equihua \& Atkinson 1987, Burgos-Solorio 1998, Equihua \& Burgos-Solorio 2002).

El objetivo del presente estudio, es proveer una lista taxonómica de las especies 
de escolitinos y platipodinos atraídas a la trampa tipo NTP-80 y proporcionar datos de distribución de las especies presentes en la región semiárida de Zapotitlán de las Salinas, Puebla.

\section{MATERIAL Y MÉTODOS}

Área de estudio. El valle de Zapotitlán de las Salinas se encuentra enclavado en la parte occidental de la reserva de la biosfera del valle de Tehuacán-Cuicatlán, al sureste del estado de Puebla y al noroeste de Oaxaca. Se localiza entre las coordenadas

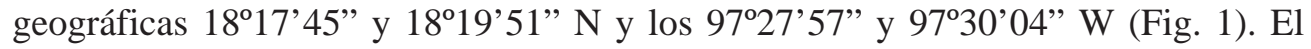
clima es de tipo semicálido (BSohw (w) (e) g) con una precipitación media anual que va de los 400 a los $450 \mathrm{~mm}$ y una temperatura media anual de $21.4^{\circ} \mathrm{C}$ (García 1988). El rango altitudinal es de 1,280 a 1,460 msnm. La vegetación está compuesta principalmente de matorral xerófilo, e intercalado se encuentran diferentes asociaciones vegetales entre los que destacan: el matorral espinoso, el mezquital, el cardonal y las cactáceas columnares (Rzedowski 1978, Oliveros 2000). La flora de la zona contiene alrededor de 3,000 especies de plantas vasculares (Rzedowski 1978, Villaseñor et al. 1990, Dávila et al. 1993, Montaña \& Valiente-Banuet 1998).

Trabajo de campo. Se realizaron colectas mensuales de abril de 1998 a marzo de 1999 por medio de 21 necrotrampas tipo NTP-80 (Morón \& Terrón 1984). Los dispositivos fueron cebados con calamar. El líquido conservador fue compuesto de 95 partes de alcohol al 70\% y cinco partes de ácido acético glacial. Las trampas fueron colocadas en cinco localidades que cubrieron las vegetaciones más representativas en la región, éstas se dispusieron de acuerdo con la extensión de cada zona y aspectos logísticos: en las cactáceas columnares se instalaron seis trampas, en el mezquital con terrazas cuatro, en el mezquital ribereño siete, en el mezquital no fragmentado tres y en la vegetación alterada una. Para conocer la ubicación de los sitios y más detalles de los mismos consultar Jiménez-Sánchez et al. (2013). Los datos mensuales de la precipitación fueron obtenidos de los registros del periodo 1981-2000 de la estación meteorológica de Zapotitlán Salinas ubicada a una altura de 1,500 msnm (SMN 2012). Trabajo de laboratorio. La identificación de los ejemplares fue realizada por el tercer autor. El material biológico fue depositado en la colección entomológica del Instituto de Fitosanidad, Colegio de Postgraduados. La distribución de las especies para México se consultó en los catálogos de Equihua \& Atkinson (1987), Equihua \& Burgos-Solorio (2002) y Burgos-Solorio \& Equihua (2007).

Análisis estadístico. Se consideró la abundancia como el número de ejemplares y la riqueza como el número de especies, estos valores se obtuvieron por sitio de colecta, por mes y para toda el área en general. La abundancia se analizó mediante una prueba de ANOVA, para esto se efectuó una transformación logarítmica $(\log 10[\mathrm{x}+1])$ de los datos, con la finalidad de homogeneizar las varianzas y verificar la normalidad. La diferencia entre el número de trampas en cada sitio de estudio, no representó un des- 


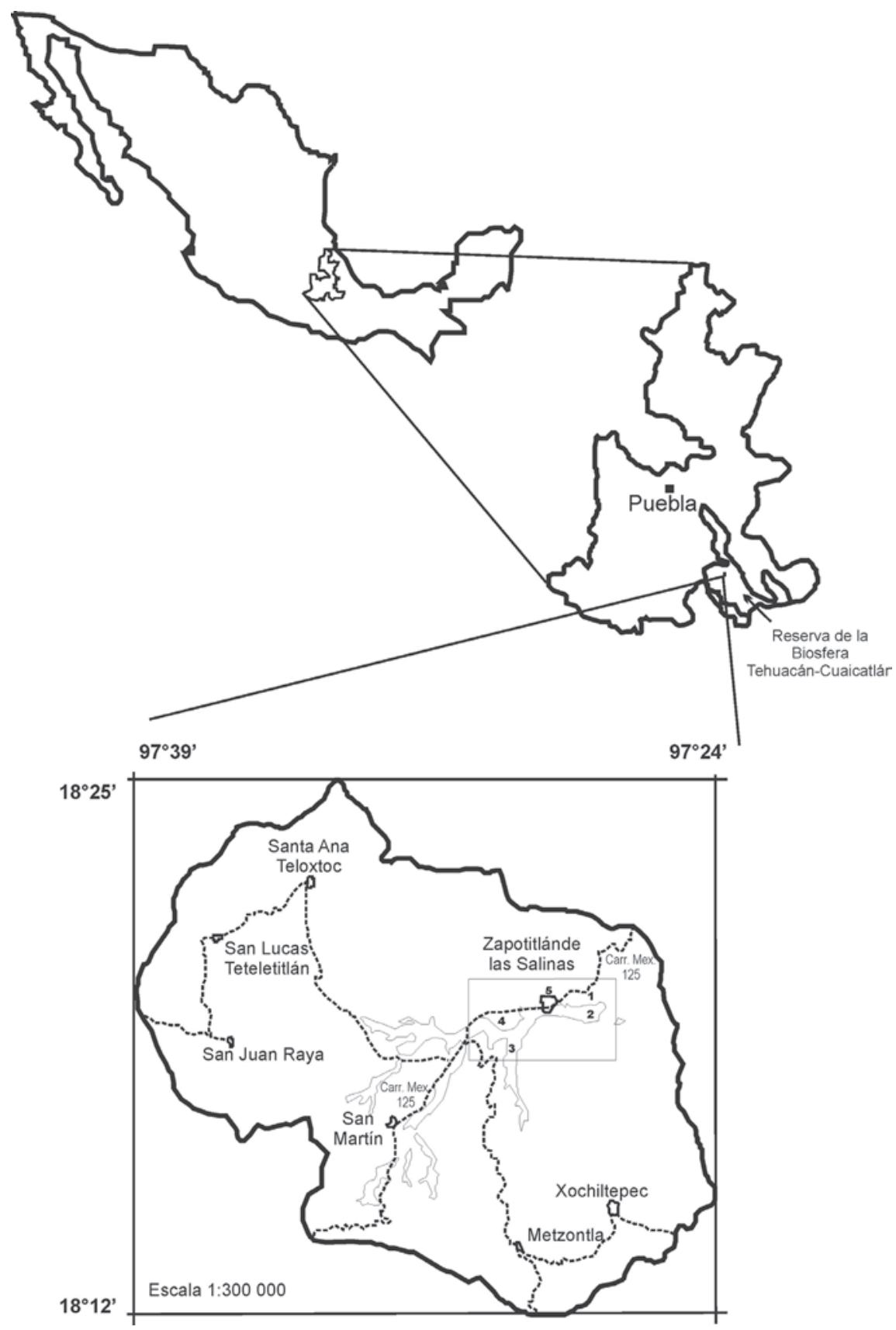

Figura. 1. Ubicación del área de estudio en Zapotitlán de las Salinas, Puebla. Figura: E. Jiménez-Sánchez. 
balance en el análisis estadístico. Las diferencias entre las medias fueron observadas a través de una prueba de Tukey (Zar 1984). Se llevó a cabo también una regresión lineal entre abundancia y la precipitación con el fin de observar si existe una relación directa con la precipitación. El tratamiento de los datos de abundancia se realizó por medio de Proc GLM y Proc REG con el programa estadístico SAS versión 9.2 (SAS Inst. 1998). Para toda el área y para cada sitio se calculó la uniformidad (E) y el índice de diversidad de Shannon (H’) con el programa PAST versión 2.12 (Hammer et al. 2001).

\section{RESULTADOS}

Se obtuvieron 328 organismos que pertenecen a cuatro géneros y seis especies (Fig. 2), de las cuales cuatro fueron registrados por primera vez para el estado de Puebla, lo que incrementa a 160 especies registradas para el estado (Cuadro 1). El género con más número de organismos fue Premnobius (162), seguido de Xyleborus (157), Monarthrum (8) y Euplatypus (1).

Las especies más abundantes en orden decreciente fueron: Premnobius cavipennis Eichhoff 1878, Xyleborus ferrugineus (Fabricius 1801), Xyleborus volvulus (Fabricius 1775) y Xyleborus posticus Eichhoff, 1869, las cuatro juntas agruparon el 97.2\% del total de organismos capturados para las dos subfamilias. Las menos abundantes fueron: Monarthrum pennatum Wood \& Bright 1992 con ocho ejemplares y Euplatypus parallelus (Fabricius 1801) con uno, ambas fueron capturadas durante la época de sequía. Esta última fue la única especie de la subfamilia Platypodinae (Cuadro 2).

La vegetación alterada presentó la menor abundancia (3) y el mezquital ribereño la mayor (264), además, este último fue diferente significativamente de las otras zonas y obtuvo todas las especies (Cuadro 2), con excepción del mezquital no fragmentado $\left(F_{4,29}=5.88, P=0.0018\right.$, Cuadro 2$)$, el cual en conjunto con el resto de las zonas no presentaron diferencias significativas.

Así mismo, el mezquital ribereño concentró todas las especies registradas y fue el único sitio donde se capturó a E. parallelus, los demás sitios no tuvieron especies exclusivas y la riqueza fue de cuatro a cinco, con excepción de la vegetación alterada donde solo se registró a $X$. volvulus. El sitio de cactáceas columnares y el mezquital no fragmentado albergaron cinco especies cada uno, a pesar de tener una abundancia baja (Cuadro 2).

Por otra parte, la diversidad para toda el área de muestreo fue de 1.3 y la uniformidad de 0.6; de manera particular la vegetación alterada tuvo un valor de diversidad de cero y para los otros cuatro sitios la diversidad fluctuó entre 1.2 y 1.3; en cuanto a la uniformidad el menor valor se obtuvo para el mezquital ribereño $(E=0.53)$ y el valor más alto correspondió a la vegetación alterada $(\mathrm{E}=1)$, para el resto de los sitios fue entre 0.7 y 0.8 (Cuadro 2). 
A

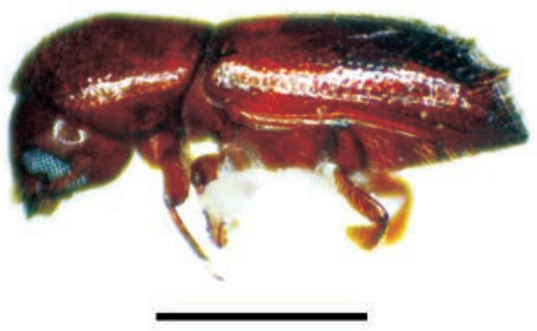

C

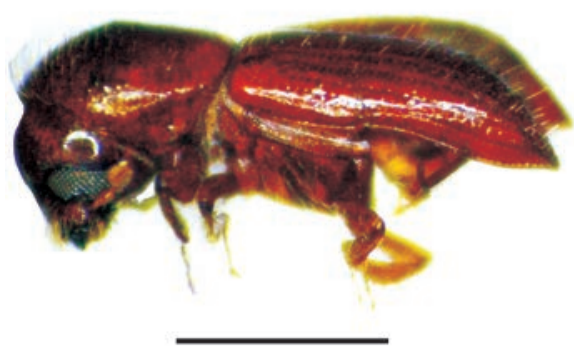

E

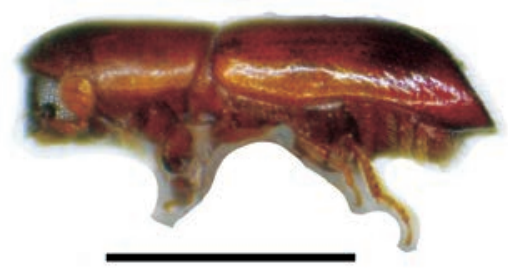

B

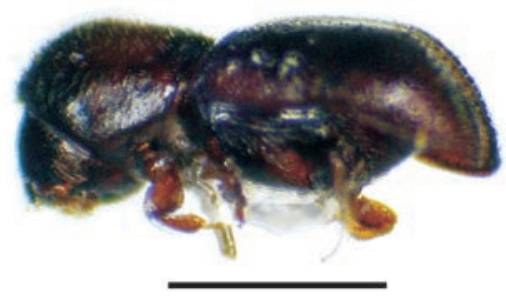

D

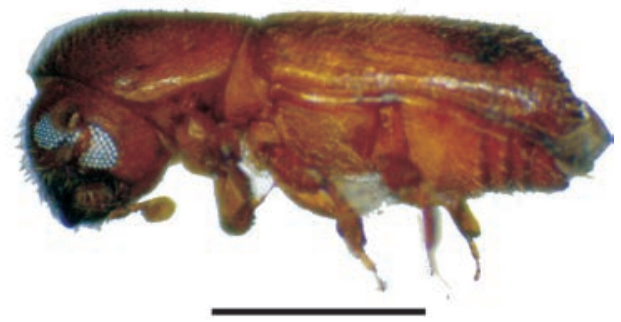

$\mathbf{F}$

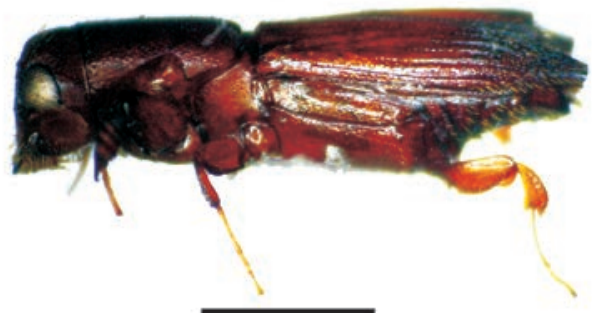

Figura 2. Vista lateral de las especies de escolitinos y platipodinos: A. Xyleborus ferrugineus. B. X.posticus. C. X. volvulus. D. Premnobius cavipennis. E. Monarthrum pennatum y F. Euplatypus parallelus. Barra de escala $=1 \mathrm{~mm}$. Fotos: E. Jiménez-Sánchez.

No se encontró una relación directa entre las abundancias de Scolytinae y la precipitación $\left(F_{1,11}=1.11, P=0.3165, r=0.1001\right)$, sus mayores abundancias fueron en el periodo de sequía.

\section{DISCUSIÓN}

El valle de Zapotitlán de las Salinas presentó una riqueza pobre de especies con respecto a otras zonas en el país, además muy pocos estados han sido estudiados 
Cuadro 1. Especies de Scolytinae y Platypodinae atraídas a trampas NTP-80 en Zapotitlán de las Salinas, Puebla. El resumen taxonómico está basado de acuerdo a Wood (1982); Wood \& Bright (1992a; 1992b) Distribución conocida en México. Abreviaturas: BC, Baja California; BCS, Baja California Sur; Camp, Campeche; Chis, Chiapas; DF, Distrito Federal; Gro, Guerrero; Hgo, Hidalgo;

Jal, Jalisco; Mex, Estado de México; Mich, Michoacán; Mor, Morelos; Nay, Nayarit; NL, Nuevo

León; Oax, Oaxaca; Pue, Puebla; QR, Quintana Roo; SLP, San Luis Potosí; Sin, Sinaloa; Son, Sonora; Tab, Tabasco; Tamps, Tamaulipas; Tlax, Tlaxcala; Ver, Veracruz; Yuc, Yucatan. ${ }^{1}$ Equihua \& Atkinson, 1987, ${ }^{2}$ Equihua-Martinez \& Burgos-Solorio (2002), ${ }^{3}$ Burgos-Solorio \& Equihua (2007). *Primeros registros para el estado de Puebla.

\begin{tabular}{|c|c|c|}
\hline CURCULIONIDAE & & \\
\hline Scolytinae & & Distribución \\
\hline Xyleborini & & \\
\hline & *Premnobius cavipennis Eichhoff, 1878 & $\begin{array}{l}\text { Chis, Camp, Gro, Mich, Mor, } \\
\text { Oax, Pue, QR, Ver, Yuc. }{ }^{2}\end{array}$ \\
\hline & Xyleborus ferrugineus (Fabricius, 1801) & $\begin{array}{l}\text { Camp, Chis, Gro, Jal, Mich, Mor, } \\
\text { Nay, NL, Oax, Pue, QR, SLP, Sin, } \\
\text { Son, Tab, Tamps, Ver., } 3\end{array}$ \\
\hline & *Xyleborus posticus Eichhoff, 1869 & $\begin{array}{l}\text { Camp, Chis, Hgo, Qro, Pue, Tab, } \\
\text { Tam, Ver. }^{2}\end{array}$ \\
\hline & Xyleborus volvulus (Fabricius, 1775) & $\begin{array}{l}\text { BCS, Camp, Chis, DF, Gro, Jal, } \\
\text { Mex, Mich, Mor, Nay, NL, Oax, } \\
\text { Pue, QR, SLP, Tab, Tamps, Ver, } \\
\text { Yuc. }{ }^{2,3}\end{array}$ \\
\hline Cothylini & *Monarthrum pennatum (Scheld, 1963) & Chis, Jal, Mich, Nay, Pue, Ver. ${ }^{2}$ \\
\hline Platypodinae & *Euplatypus parallelus (Fabricius, 1801) & $\begin{array}{l}\text { BCS, Camp, Chih, Chis, Gro, Jal, } \\
\text { Mor, Nay, Oax, Qro, Pue, QR, } \\
\text { SLP, Sin, Son, Tab, Tams, Ver, } \\
\text { Yuc. }\end{array}$ \\
\hline
\end{tabular}

(Equihua \& Burgos-Solorio 2002). Cuatro de las seis especies capturadas fueron los primeros registros para el estado. La presencia de estos organismos en las zonas áridas de México ofrece la oportunidad para incrementar el conocimiento de esta fauna de hábitos xilomicetófagos, sobre todo porque este grupo se encuentra normalmente en zonas tropicales y templadas (Equihua 1992, Equihua \& Burgos-Solorio 2002).

Por otro lado, la escasa cobertura vegetal y las condiciones climáticas extremas que caracterizan al tipo semicálido como son tener una temperatura media anual de $21^{\circ} \mathrm{C}$, una precipitación anual de $400 \mathrm{~mm}$ y una canícula bien definida de marzo a mayo (García 1988) son una limitante para el establecimiento de las especies en esta zona, estos factores podrían explicar la baja abundancia de las especies, sin embargo, hubo una alta dominancia de dos especies que concentraron el 73\% del total de organismos. Este fenómeno se observa de igual manera en las especies necrófilas donde 
Cuadro 2. Abundancia y riqueza de las especies capturadas en cada uno de los sitios de muestreo, así como, los valores de diversidad y uniformidad de Shannon. Las letras iguales "a” o "b” al lado del valor de la abundancia indican que no hay diferencias significativas entre los sitios, mientras que las letras diferentes muestran diferencias significativas entre éstos con la prueba de Tukey $(P<0.0001)$.

\begin{tabular}{lcccccc}
\hline & $\begin{array}{c}\text { Cactáceas } \\
\text { columnares }\end{array}$ & $\begin{array}{c}\text { Mezquital } \\
\text { terrazas }\end{array}$ & $\begin{array}{c}\text { Mezquital } \\
\text { ribereño }\end{array}$ & $\begin{array}{c}\text { Mezquital no } \\
\text { fragmentado }\end{array}$ & $\begin{array}{c}\text { Vegetación } \\
\text { alterada }\end{array}$ & Total \\
\hline Premnobius cavipennis & 2 & 0 & 149 & 11 & 0 & 162 \\
Xyleborus ferrugineus & 4 & 2 & 65 & 6 & 0 & 77 \\
Xyleborus volvulus & 9 & 7 & 23 & 12 & 3 & 54 \\
Xyleborus posticus & 1 & 2 & 22 & 1 & 0 & 26 \\
Monarthrum pennatum & 1 & 2 & 4 & 1 & 0 & 8 \\
Euplatypus parallelus & 0 & 0 & 1 & 0 & 0 & 1 \\
Abundancia & $17 \mathbf{b}$ & $13 \mathbf{b}$ & $264 \mathbf{a}$ & $31 \mathbf{a b}$ & $3 \mathbf{b}$ & 328 \\
Riqueza & 5 & 4 & 6 & 5 & 1 & 6 \\
Índice de diversidad & 1.26 & 1.19 & 1.17 & 1.27 & 0 & 1.29 \\
Uniformidad & 0.7 & 0.82 & 0.53 & 0.71 & 1 & 0.6 \\
\hline
\end{tabular}

dos especies agruparon el 86\% de la abundancia total (Jiménez-Sánchez et al. 2013). A pesar de que no se obtuvieron datos más precisos sobre la vegetación y el clima, este trabajo deja entre ver, que estos factores ambientales podrían tener un papel importante en la biología de estos grupos, por lo que se tendría que explorar en futuras investigaciones.

La mayor abundancia que se presentó en el mezquital ribereño y el mezquital no fragmentado, quizá se debió a la presencia de más especies leñosas en estos sitios (Oliveros 2000), lo cual ayudó al desarrollo y el establecimiento de las especies capturadas que se sabe son xilomicetófagas (Atkinson \& Equihua 1986, Equihua \& Atkinson 1986, Beaver 1988, Zanuncio et al. 2005, Burgos-Solorio \& Equihua 2007). Las poblaciones de escolitinos encontraron las condiciones para su establecimiento principalmente en el mezquital ribereño, donde se tuvo la presencia de un río con flujo de agua pequeño pero constante, aunado a la mayor cobertura vegetal y el desarrollo de las actividades productivas del lugar (Jiménez-Sánchez et al. 2013). De tal forma que los factores como la fisiografía, el micro clima, la diversidad de plantas hospederas y la biología de las especies pudieron haber tenido un papel fundamental, que permitió la mayor concentración de organismos y el agrupamiento de todas las especies en estos lugares. Mientras que los otros sitios, presentaron más especies de cactáceas, o estuvieron perturbadas, por lo que la cobertura vegetal fue menor y las condiciones de humedad y temperatura fueron más extremas, de tal manera que la presencia de estos escarabajos posiblemente se debió a que son lugares por donde transitan en la búsqueda de hospederos. 
Las mayores poblaciones de escolitinos y platipodinos estuvieron presentes después de la época de lluvias, por lo que este no fue un factor determinante (Fig. 3). Estos dos grupos se alimentan principalmente de hongos que generalmente inoculan en árboles maduros, por lo que posiblemente su presencia está posiblemente asociada a otros factores como: el periodo de maduración de los hongos que inoculan y se alimentan, una acumulación de la humedad en la materia en descomposición, periodos reproductivos cuando el recurso puede ser más apto para el buen desarrollo de las larvas, posiblemente la lluvia limita el vuelo que es muy importante para realizar la infestación de nuevos hospederos, cabe señalar que son hipótesis que podrían representar un gran interés en futuras investigaciones en este grupo y en esta zona.

El patrón fenológico mencionado coincide con los encontrados en una región de Nayarit por Soto et al. (2013), otra región en el estado de Tabasco por Pérez de la Cruz et al. (2011) y en la zona de estudio por Jiménez-Sánchez et al. (2013). Parece haber una asociación con la disponibilidad de los recursos vegetales generados después de las lluvias y por los elementos vegetales del matorral xerófilo como la floración y fructificación que ocurren al final de dicho periodo (Arias et al. 2000, Pérez de la Cruz et al. 2011). La abundancia y riqueza de especies de Scolytinae y Platypodinae en Zapotitlán de las Salinas, podrían indicar una alta riqueza específica y un alto endemismo en esta zona árida del país, si se considera que las especies de escolitinos y platipodinos obtenidas están asociadas principalmente a climas más tropicales y con alta humedad (Equihua \& Atkinson 1986, Equihua 1992, Equihua \& Atkinson 1987), sin embargo, es necesario realizar estudios faunísticos sistemáticos en otras regiones áridas del país.

La trampa NTP-80 es un método eficiente para la captura de insectos necrófilos,

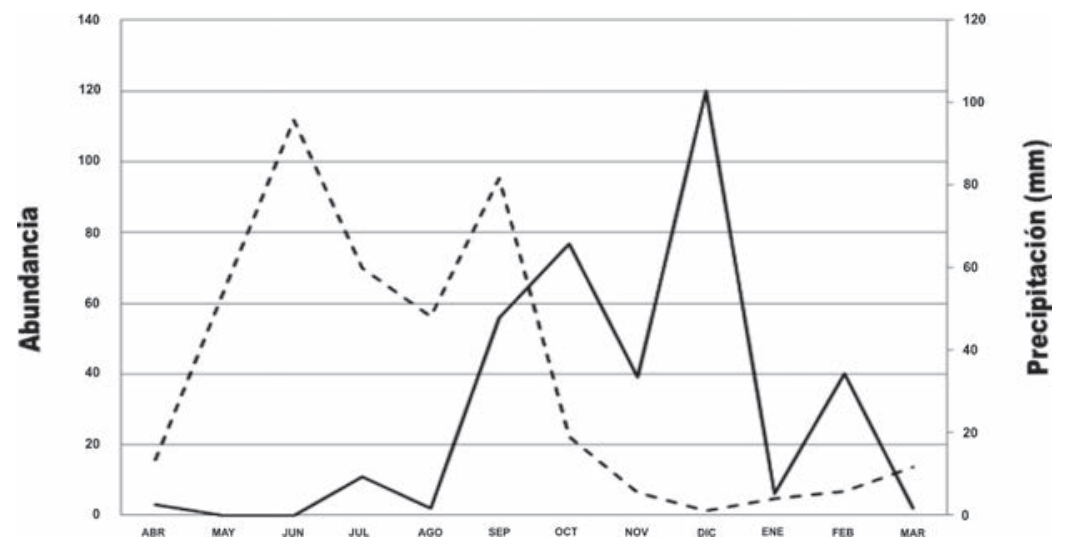

Figura 3. Abundancia mensual de Scolytinae (línea continua) y su relación con la precipitación (línea punteada). 
sin embargo, los escolitinos y platipodinos se ven atraídos por el uso de alcohol etílico (Moeck 1970, Wood 1982) usado como líquido conservador, lo cual ha permitido estudios de estos grupos de forma colateral con el empleo de este tipo de trampas (Equihua 1992, Burgos-Solorio 1998, Muñiz 1998).

AGRADECIMIENTOS. Este estudio fue financiado parcialmente por el Proyecto Zoología de la División de Investigación y Posgrado de la FES-Iztacala, UNAM. Los autores agradecen al Dr. Álvaro Fuentealba por sus comentarios en el análisis estadístico.

\section{LITERATURA CITADA}

Arias, T. A. A., Valverde, M. T. \& Reyes, S. J. 2000. Las plantas de la región de Zapotitlán de las Salinas, Puebla. INE, Red para el Desarrollo Sostenible, A. C. Universidad Nacional Autónoma de México, D.F., México.

Atkinson, T. H., \& Equihua, A. 1986. Biology of bark and ambrosia beetles (Coleoptera: Scolytidae and Platypodidae) of a tropical rain forest in southeastern Mexico with an annotated checklist of species. Annals of the Entomological Society of America, 79: 414-423.

Beaver, R. A. 1988. Biological studies on ambrosia beetles of the Seychelles (Col., Scolytidae and Platypodidae). Journal of Applied Entomology, 105: 62-73.

Burgos-Solorio, A. 1998. Escarabajos barrenadores Platypodidae y Scolytidae (Coleoptera) atraidos a trampas NTP-80 de la Cañada de los alrededores de San José de los Laureles, Tlayacapan, Morelos, Mexico. Dugesiana, 5: 529-534.

Burgos-Solorio, A., \& Equihua, A. 2007. Platypodidae y Scolytidae (Coleoptera) de Jalisco, México. Dugesiana, 14: 59-82.

Casas, A., Valiente-Banuet, A., Viveros, J. L., Caballero, J., Cortés, L., Dávila, P., Lira, R. \& Rodríguez, I. 2001. Plant resources of the Tehuacán-Cuicatlán valley, Mexico. Economic Botany, 55: 129-166.

Cejudo-Espinosa, E. \& Deloya, C. 2005. Coleoptera necrófilos del bosque de Pinus hartwegii del Nevado de Toluca, México. Folia Entomológica Mexicana, 44: 67-73.

Dávila, P., Villaseñor, J. L., Medina, L., Ramírez, A., Salinas, A., Sánchez-Ken, J. \& Tenorio, P. 1993. Flora del Valle Tehuacán-Cuicatlán. Listados Florísticos de México X. México: Instituto de Biología, Universidad Nacional Autónoma de México, D.F., México.

Dávila, P., Arizmendi, M. del C., Valiente-Banuet, A., Villaseñor, J. S., Casas, A. \& Lira, S. R. 2002. Biological diversity in the Tehuacán-Cuicatlán Valley, Mexico. Biodiversity and Conservation, 11: 421-442.

Deloya, C., Ruíz-Lizárraga, G. \& Morón, M. A. 1987. Análisis de la entomofauna necrófila de la Región de Jojutla, Morelos, México. Folia Entomológica Mexicana, 73: 157-171.

Equihua, M. A. 1992. Coleópteros Scolytidae atraídos a trampas NTP-80 en el Soconusco, Chiapas, México. Folia Entomológica Mexicana, 84: 55-66.

Equihua, M. A., \& Atkinson, T. H. 1986. Annotated checklist of bark and ambrosia beetles (Coleoptera, Scolytidae and Platypodidae) associated with a tropical decidious forest at Chamela, Jalisco, Mexico. The Florida Entomologist, 69: 619-628.

Equihua, M. A. \& Atkinson, T. H. 1987. Catálogo de platypodidae (Coleoptera) de Norte y Centroamérica. Folia Entomológica Mexicana, (72): 5-31.

Equihua, M., A. \& Burgos-Solorio, A. 2002. Scolytidae. pp. 539-557. In: J. E. Llorente-Bousquets y J. J. Morrone (Eds.). Biodiversidad, Taxonomía y Biogeografía de Artrópodos de México: Hacia una 
síntesis de su conocimiento. Vol. III. CONABIO IBUNAM. México, D. F.

García, E. 1988. Modificaciones al sistema de clasificación climática de Koppen: para adaptarlo a las condiciones de la República Mexicana. Editado por el Autor, México.

Gennard, D. E. 2007. Forensic entomology. An introduction. John Wiley \& Sons, West Sussex, England.

Hammer, Ø., Harper, D. A. T. \& Ryan, P. D. 2001. PAST: Paleontological Statistics Software Package for Education and Data Analysis. Paleontologia Electronica, 4: 10-50.

Jiménez-Sánchez, E., Quezada-García, R. \& Padilla-Ramírez, J. 2013. Diversidad de escarabajos necrófilos (Coleoptera: Scarabaeidae, Silphidae, Staphylinidae y Trogidae) en una región semiárida del valle de Zapotitlán de las Salinas, Puebla, México. Revista de Biología Tropical, 61: 14751491.

Moeck, H. A. 1970. Ethanol as the primary attractant for the ambrosia beetle Trypodendron lineatum (Coleoptera: Scolytidae). The Canadian Entomologist, 102: 985-995.

Montaña, C. \& Valiente-Banuet, A. 1998. Floristic and life-form diversity along an altitudinal gradient in an intertropical semiarid Mexican region. The Southwest Naturalist, 43: 25-39.

Morón, M. A. \& López-Méndez, J. A. 1985. Análisis de la entomofauna necrófila de un cafetal en el Soconusco, Chiapas, México. Folia Entomológica Mexicana, 63: 47-59.

Morón, M. A. \& Terrón, R. A. 1982. Análisis de la entomofauna necrófila de la cañada de Otongo, Hidalgo. Folia Entomológica Mexicana, 53: 38-39.

Morón, M. A. \& Terrón, R. A. 1984. Distribución altitudinal y estacional de los insectos necrófilos en la Sierra Norte de Hidalgo, México. Acta Zoológica Mexicana, (n.s.) 3: 1-47.

Morón, M. A., Camal, J. F. \& Canul, O. 1986. Análisis de la entomofauna necrófila del área norte de la Reserva de la Biosfera “Sian Ka’an”, Quintana Roo, México. Folia Entomológica Mexicana, 69: 83-98.

Muñiz, V. R. 1998. Curculionoidea recolectados en necrotrampas. Dugesiana, 5: 1-9.

Navarrete-Heredia, J. L., Sainz, C. I., González-Hernández, A. L., Quiroz-Rocha, G. A., Hernández, A., Vásquez-Bolaños, M., Vega-Romero, D. \& Hernández, B. 2012. Coleópteros necrófilos del bosque de Los Colomos, Guadalajara, Jalisco, México. Dugesiana, 19: 157-164.

Oliveros, O. 2000. Descripción estructural de las comunidades vegetales en las terrazas fluviales del río El Salado, en el Valle de Zapotitlán de las Salinas, Puebla, México. Tesis de licenciatura, Universidad Nacional Autónoma de México, Estado de México, México.

Paredes-Flores, M., Lira, R. S. \& Dávila, P. D. A. 2007. Estudio etnobotánico de Zapotitlán de las Salinas, Puebla. Acta Botánica Mexicana, (79): 13-61.

Pérez de la Cruz, M., Valdéz, J. M. C., Nápoles, J. R., Equihua, M. A., Sánchez, S. S. \& de la Cruz, P. A. 2011. Fluctuación poblacional, plantas huéspedes distribución y clave para la identificación de platypodinae (Coleoptera: Curculionidae) asociados al agroecosistema cacao en Tabasco México. Acta Zoológica Mexicana, (n.s.) 27: 129-143.

Rzedowski, J. 1978. Vegetación de México. Limusa, D.F. México.

SAS Institute Inc. 1998. SAS user's guide; statistics 1998. SAS Institute Cary North Carolina. EE.UU. Servicio Meteorológico Nacional (SMN). 2012. Climatología. Normales climatológicas por estación. Comisión Nacional del Agua, México. (Consultado: 9 febrero 2013, http://smn.cna.gob.mx/index. php?option=com_content\&view=article\&id=42\&Ite id=28).

Soto, H. M., Martínez, O. G. \& Carbajal, C. C. 2013. Fauna de Curculionidae (Coleoptera) en huertas de aguacate Hass (Persea americana Mill) en Xalisco, Nayarit. Dugesiana, 20: 93-98.

Villaseñor, J. L., Dávila, P. \& Chiang, F. 1990. Fitogeografía del Valle de Tehuacán-Cuicatlán. Boletín de la Sociedad Botánica de México, 50: 135-149.

Wood, S. L. 1982. The bark and ambrosia beetles of North and Central America (Coleoptera: Scolyti- 
dae), a Taxonomic Monograph. Great Basin Naturalist Memoirs, 6: 1-1359.

Wood, S. L. \& Bright, D. E. 1992a. A catalog of Scolytidae and Platypodidae (Coleoptera), Part 2: Taxonomic Index. Great Basin Naturalist Memoirs, Vol. A: 1-833.

Wood, S. L. \& Bright, D. E. 1992b. A catalog of Scolytidae and Platypodidae (Coleoptera), Part 2: Taxonomic Index. Great Basin Naturalist Memoirs, Vol. B: 835-1553.

Zanuncio, J. C., Sossai, M. F., Flechtmann, C. A. H., Zanuncio, T. V., Guimarães, E. M. \& Espindula, F. C. 2005. Plants of a Eucalyptus clone damaged by Scolytidae and Platypodidae (Coleoptera). Pesquisa Agropecuária Brasileira, 40: 513-515.

Zar, J. H. 1984. Biostatiscal Analysis. Prentice Hall, Eaglewood, EE.UU. 